of smaller marker panels, we have developed anti-MVP into prototypic bead-based ELISA format.

Results Discovery and validation experiments using the NavigAID SLE array showed that anti-MVP antibodies occurred with frequencies of $15 \%-30 \%$ in three different SLE cohorts at a specificity of 97\%. Exploratory testing of multi-marker panels consisting of anti-MVP in combination with antidsDNA, anti-ribosomal $\mathrm{P}$ and anti-SmD yielded a $6 \%$ increase in sensitivity at $98 \%$ without loss of specificity. Multivariate data projection methods revealed that anti-MVP is detected in a subset of SLE patients with little overlap to established marker..A bead-based ELISA was developed for measuring anti-MVP antibodies and showed good correlation with Luminex data $(\mathrm{R}=0.88)$ indicating successful platform transfer.

Conclusions Anti-MVP autoantibodies represent a useful marker in SLE and, in combination with established markers, optimises the strategy for autoantibody testing. Furthermore, although more studies are needed, our findings suggest a previously undescribed linkage of type I IFN and autoantibody targets in SLE.

\section{AUTOANTIBODIES DICTATE CLINICAL MANIFESTATIONS IN PATIENTS WITH SYSTEMIC LUPUS ERYTHEMATOSUS}

${ }^{1,2}$ A Burlui* ${ }^{1,2}$ A Cardoneanu, ${ }^{1,2}$ E Rezus. ' Gr T Popa University of Medicine and Pharmacy, Rheumatology, lasi, Romania; ${ }^{2}$ Clinical Rehabilitation Hospital, Rheumatology, lasi, Romania

\subsection{6/lupus-2017-000215.268}

Background and aims Systemic Lupus Erythematosus (SLE) is known for its multifaceted clinical features and complex immune disturbance. Numerous studies have proven that certain autoantibodies are linked to specific clinical manifestations. However, the diversity of possible associations makes for the uniqueness of each case of SLE. The goal of our study was to analyse the link between clinical presentation and autoantibody titers in Romanian patients with SLE.

Methods We conducted an observational study of 48 adult patients with SLE hospitalised in the Rheumatology Department of the Clinical Rehabilitation Hospital. Venous blood samples were drawn to measure antinuclear antibody levels as well as anti-dsDNA, anti-ssDNA, anti-Sm, anti-U1RNP, antiSSA, anti-SSB and anti-nucleosome antibody titers (ELISA). Clinical presentation, biochemical tests, SLEDAI score values and urinalysis were extracted from patients' charts. Patient characteristics were included in a database and analysed using IBM SPSS Statistics v20.

Results We found statistically significant correlations $(\mathrm{p}<0.05)$ between cutaneous manifestations and anti-Sm, anti-U1RNP, anti-SSA, anti-SSB and anti-nucleosome antibodies. Kidney involvement correlated with anti-Sm, anti-U1RNP and antinucleosome antibodies $(\mathrm{p}<0.05)$. Joint involvement was strongly associated with the presence of anti-U1RNP antibodies $(p=0.001)$. Haematological abnormalities were significantly correlated with anti-dsDNA, anti-U1RNP, anti-SSA and antiSSB antibodies $(p<0.05)$, while ESR and CRP levels were only associated with anti-U1RNP antibodies $(p=0.03)$. Furthermore, SLEDAI scores correlated with anti-dsDNA and antinucleosome antibody titers $(\mathrm{p}<0.05)$.

Conclusions Our data support the relationship between autoantibody titers, disease activity and severity of clinical changes in Romanian patients with systemic lupus erythematosus.

\section{SINGLE NUCLEOTIDE POLYMORPHISMS (SNPS) OF INTEGRIN-ALPHA-M (ITGAM) ARE ASSOCIATED WITH LUPUS NEPHRITIS (LN) IN AN ASIAN SYSTEMIC LUPUS ERYTHEMATOSUS (SLE) COHORT}

${ }^{1} \mathrm{M}$ Chan ${ }^{*},{ }^{1}$ WG Law, ${ }^{1} \mathrm{TY}$ Lian, ${ }^{1} \mathrm{KO}$ Kong, ${ }^{2} \mathrm{CY}$ Yu, ${ }^{3} \mathrm{YW}$ Song, ${ }^{1} \mathrm{HH}$ Chng, ${ }^{1} \mathrm{~B}$ Leung, ${ }^{4} \mathrm{~B}$ Tsao, ${ }^{1} \mathrm{HS}$ Howe. 'Tan Tock Seng Hospital, Rheumatology Allergy and Immunology, Singapore, Singapore; ${ }^{2}$ The Research Institute at Nationwide Children's Hospital, Centre for Molecular and Human Genetics, Columbus, USA; ${ }^{3}$ Seoul National University, Rheumatology, Seoul, Republic of Korea; ' University of California Los Angeles, Rheumatology, Los Angeles, USA

\subsection{6/lupus-2017-000215.269}

Background The Integrin-alpha-M (ITGAM) rs1143679 SNP has been associated with susceptibility to SLE and lupus nephritis (LN) in oriental Chinese and Thai populations. We previously found 13 ITGAM SNPs in linkage disequilibrium (LD) that were associated with susceptibility to SLE, but found no association with rs1143679.

Aim To determine associations of ITGAM SNPs with SLE subphenotypes and autoantibodies.

Methods We studied 248 patients fulfilling the 1997 ACR revised criteria for SLE. SLE-associated ITGAM SNP alleles were identified using custom-designed Immunochip arrays and gPLINK 1.062 software, with Bonferroni corrections for multiple comparisons. Associations of SLE-related ITGAM SNPs with SLE subphenotypes (malar or discoid rash, serositis, mouth ulcers, arthritis, haematological, renal or neurological involvement) and autoantibodies to dsDNA, Ro, RNP or Sm were determined with chi-square and Fisher's tests and logistic regression.

Results All 13 SLE susceptibility ITGAM SNPs as well as the uncommon rs1143679 SNP $(n=11)$ were associated with LN (Table 1). The strongest association was with rs2359661 $(p=0.002$, uncorrected). Subjects with these SNPs were less likely to have discoid rash. There was a trend towards an association with anti-Sm. Logistic regression models for 11 SNPs retained the factors LN, discoid rash and anti-Sm, suggesting strong LD for these SNPs.

Conclusions This study demonstrated novel ITGAM SNP associations with LN and confirmed the association of rs1143679 with LN. Most associated SNPs were in the regulatory region of ITGAM bearing promoter/enhancer histone marks and have been associated with expression levels in several cell types, suggesting modulation of levels of ITGAM expression to impact these subphenotypes.

\section{ASSOCIATION OF TLR2 (23BP INS/DEL) POLYMORPHISM WITH SYSTEMIC LUPUS ERYTHEMATOSUS (SLE) AND P. FALCIPARUM MALARIA: A STUDY IN MALARIA ENDEMIC AREA OF ODISHA, INDIA}

${ }^{1}$ B Das*, ${ }^{2}$ R Tripathy, ${ }^{1} S$ Pattanaik, ${ }^{3}$ A Panda. ${ }^{1}$ S. C.B. Medical College, Medicine, Cuttack, India; ${ }^{2}$ S.C.B. Medical College, Biochemistry, Cuttack, India; ${ }^{3}$ Central University of Jharkhand, Centre for Life Sciences, Ranchi, India

\subsection{6/lupus-2017-000215.270}

Background and aims Human toll-like receptors (TLRs) participate in the innate response and signal the activation of adaptive immunity. TLRs play a vital role in sensing infection. A common $23 \mathrm{bp}$ insertion/deletion polymorphism at 5'UTR of TLR2 gene has been shown to affect TLR2 expression and plasma levels of pro-inflammatory molecules. We hypothesised 